\title{
Relationship between lipid accumulation product index and arterial stiffness in the Chinese population with hypertension: a report from the China Hypertension Registry Study
}

\section{Yumeng Shi}

Nanchang University Second Affiliated Hospital

Lihua Hu

Peking University First Hospital

Minghui Li

Nanchang University Second Affiliated Hospital

\section{Wei Zhou}

Nanchang University Second Affiliated Hospital

\section{Tao Wang}

Nanchang University Second Affiliated Hospital

\section{Lingjuan Zhu}

Nanchang University Second Affiliated Hospital

\section{Huihui Bao}

Nanchang University Second Affiliated Hospital

\section{Ping Li}

Nanchang University Second Affiliated Hospital

Xiaoshu Cheng ( $\nabla$ xiaoshumenfan126@163.com )

Nanchang University Second Affiliated Hospital

\section{Original investigation}

Keywords: Lipid accumulation product index, Brachial-ankle pulse wave velocity, Arterial stifness, Hypertension

Posted Date: August 24th, 2021

DOl: https://doi.org/10.21203/rs.3.rs-820744/v1

License: (c) (1) This work is licensed under a Creative Commons Attribution 4.0 International License. Read Full License 
Version of Record: A version of this preprint was published at Frontiers in Cardiovascular Medicine on January 21st, 2022. See the published version at https://doi.org/10.3389/fcvm.2021.760361. 


\section{Abstract}

Background: The relation of LAP index with baPWV have been evaluated in several previous studies and yield inconsistent results. The aim of the study was to investigate the association between the lipid accumulation product (LAP) index and arterial stiffness in the Chinese population with hypertension.

Methods: We conducted a cross-sectional analysis in 4926 participants chinese population with hypertension (aged $64.42 \pm 9.44$ years). The LAP was developed from population-based frequency plots of adult waist circumferences and circulating triglyceride concentrations. Arterial stifness was determined by measuring brachial-ankle pulse wave velocity (baPWV).

Results: Multivariate linear regression analyses showed that LAP index was independently and positively associated with baPWV $(\beta, 25.06 \mathrm{~cm} / \mathrm{s}$ (95\% confdence interval [Cl]: 10.91, $39.22 \mathrm{~cm} / \mathrm{s})$. Consistently, Multiple logistic analyses showed a positive association between LAP index risk of elevated baPWV (>75th percentile) (odds ratio [OR], 1.24; 95\% $\mathrm{Cl} 1.09,1.40$ ). A restricted cubic spline showed that the significant linear association existed between LAP index and baPWV. Similar results were found in various subgroups.

Conclusion: LAP is a signifcant positive associated with with baPWV and elevated baPWV in Chinese hypertensive adults.

\section{Background}

The European Society of Hypertension/European Society of Cardiology Guidelines on Arterial Hypertension suggest that brachial-ankle pulse wave velocity (baPWV) measurement should be used as a tool to evaluate subclinical damage of target organs[1]. So, arterial stiffness can be evaluated noninvasively by measuring baPWV, which is the gold standard and widely used method. It is reported that metabolic syndrome[2], cardiovascular disease(CVD)[3], stroke[4], mortality[5], and kidney disease[6] are all related to elevated baPWV. In China, hypertension is not only a common health problem but also a major pathogenic factor of arterial stiffness, which can cause a high proportion of hypertension and cardiovascula disability $[7,8]$. According to the latest national hypertension survey of 451,755 adults in China from 2012 to 2015, the prevalence rate of hypertension is $27.9 \%[9]$. There are estimates suggesting that the total number of hypertension patients among Chinese adults is 244.5 million[10]. Therefore, a better understanding of the potential risk factors of increased arterial stiffness among hypertensive patients may help to prevent the onset of arterial stiffness and related cardiovascular diseases.

Lipid accumulation product (LAP) index, as a new index to predict cardiovascular diseases, has attracted the attention of many researchers. LAP was proposed as a better continuous marker/index to describe lipid over-accumulation in relationship to central obesity and metabolic risks[11], and was shown to outperform BMI in the identification of CVD risk[12] and diabetes[13]. The calculation method of LAP index varies with gender: males: [(WC-65) * TG]; women: [(WC-58) *TG][14]. There are only a few previous studies on LAP and arterial stiffness, but their conclusions are inconsistent[15-17]. Moreover, all the 
above studies were carried out in healthy adults, and few people in hypertensive patients pay attention to the effect of LAP on arterial stiffness.

In order to fill the gap in the above research. This cross-sectional analysis uses the data of a Chinese hypertension registration study to evaluate the association between the LAP index and arterial stiffness in the Chinese population with hypertension.

\section{Methods}

\section{Subject population and design}

This study was based on the data collected by the China hypertension Registry Study (Registration number: ChiCTR1800017274); Briefly, the China Hypertension Registry Study is an ongoing, real-world, observational study conducted in Wuyuan, Jiangxi province of China. The full details regarding the design and rationale of the study were extensively described elsewhere[18]. Participants were recruited in 2018 were consecutively enrolled if they (1) were aged $\geq 18$ years, (2) had hypertension, defined as seated resting blood pressure $\geq 140 / 90 \mathrm{mmHg}$ or self-reported use of antihypertensive medications. The participants were excluded when subjects with neurological abnormalities led to the inability to follow-up according to the study protocol. In accordance with the Declaration of Helsinki conducted the study. Approval of the study protocol was provided by the Ethics Committees of the Biomedical Institute of Anhui Medical University (Approved No. Of ethic committee: CH1059). Before each participant entered this study, they all signed informed consent.

A total of 5233 subjects had baPWV measurements taken at baseline. After excluding those with anklebrachial systolic blood pressure indexes $(A B I)<0.90(n=133)$, or using lipid-lowering medications $(n=$ 174), Finally, 4926 patients were selected for the study (Figure S1).

\section{Data Collection}

Standardized questionnaires were used to obtain information about demographic factors, lifestyle behaviours (smoking and alcohol consumption), disease history, family history of diseases, and medication use at baseline. At the same time, we also collected the anthropometric indices included weight, height, waist circumference (WC), hip circumference (HC) of all participants. The body mass index (BMI) was calculated as the body weight in kilograms / square of the height in meters $\left(\mathrm{kg} / \mathrm{m}^{2}\right)$. Blood pressure (BP) was measured in the sitting position using an electronic sphygmomanometers (Omron; Dalian, China). After a 10-minute rest period, BP was measured three times, and the average of the three measurements was used for final analyses.

\section{BaPWV measurements}


The baPWV $(\mathrm{cm} / \mathrm{s})$ and ankle-brachial index $(A B I)$ was automatically measured simultaneouslyin the supine position after having rested for more than 10 minutes, using automatic waveform analyzer ( BP203RPE III device; Omron Health Care, Kyoto, Japan). The specific measurement method is as follows: blood pressure cuff is wrapped on arms and ankles, the lower edge of the armband is located $2-3 \mathrm{~cm}$ above the cubital fossa transverse stripes, while the lower edge of the ankle band is located $1-2 \mathrm{~cm}$ above the medial malleolus. And the baPWV value was calculated as the ratio of transmission distance from the brachium to ankle divided by the transit time:. Lb and La refer to the path length from suprasternal incision to brachial muscle and from suprasternal incision to ankle (La), respectively. $\Delta \mathrm{Tba}$ is the ankle-brachial artery pressure wave foot time difference. baPWV was measured twice automatically and the higher value of the the left and right side was used in the final analysis.

\section{Laboratory Tests}

After an 8-10hour fasting period, all blood samples were taken from the cubital vein and delivered to the Biaojia Biotechnology Laboratory, Shenzhen, China. Automatic clinical analyzers (Beckman Coulter) were used to measure biochemical included creatinine, uric acid, plasma homocysteine (Hcy), fasting total cholesterol (TC), triglycerides (TG), and high-density lipoprotein cholesterol (HDL-C), and fasting plasma glucose $(F P G)$. LAP was determined from WC $(\mathrm{cm})$ plus TG $(\mathrm{mmol} / \mathrm{L})$ for men $[(\mathrm{WC}-65)$ * $\mathrm{TG}]$ and women [(WC-58) *TG][19]; To prevent non-positive values for LAP, any male WC values of $65 \mathrm{~cm}$ or less were revised upward to $66.0 \mathrm{~cm}$, and any female WC values of $58 \mathrm{~cm}$ or less were revised upward to $59 \mathrm{~cm}[12]$. The estimated glomerular filtration rate (eGFR) was calculated by the chronic kidney disease (CKD) epidemiology collaboration formula[20].

\section{Statistical Analysis}

Based on quartiles of LAP levels, the study population was divided into four groups. The characteristics were presented as mean (SD), and categorical variables were expressed as count (percentage). We compared baseline characteristics on participants quartiles of LAP levels by one-way analysis of variance (ANOVA) for continuous variables and chi-square or Fisher's exact test for categorical variables, as appropriate. The distribution of serum concentrations of TBiL was strongly skewed toward the left. Thus, we performed the In-transformed (In LAP) before analysis.

Because the predictive value of baPWV in cardiovascular events is unavailable. In this study, elevated baPWV is defined as a value greater than $75 \%$ of baPWV value and greater than $20.59 \mathrm{~cm} / \mathrm{s}$. Beta coefcient $(\beta)$ and $95 \%$ confidence interval $(\mathrm{Cl})$ used to investigate the association between LAP and baPWV were calculated using multivariate linear regression; Odds ratios (OR) and $95 \% \mathrm{Cl}$ used to investigate the association between LAP and elevated baPWV in hypertensive participants were calculated using multivariate logistic regression for three models. These three models are as follows: model 1 adjusted only for age, sex; model 2 adjusted for prior covariates and BMI, SBP, DBP, pulse, smoking status, drinking status, model 3 adjusted for these prior covariates plus FBG, TC, HDL, LDL-C, Hcy, uric acid, eGFR, diabetes mellitus, antihypertensive drugs, antiplatelet drugs. Variables known as traditional risk factors for stroke and the potential confounders were selected if the effect estimates 
individually changed by at least $10 \%[21]$. To characterize the shape of the dose-response relationship of LAP index with baPWV and elevated baPWV, we performed a generalized additive model (GAM) and a fitted smoothing curve (penalized spline method). As additional exploratory analyses, possible modifications of the association between LAP index and baPWV were also evaluated for variables including sex (male vs. Female), age ( $<65 \mathrm{vs} . \geq 65$ years), BMl ( $<25 \mathrm{vs} . \geq 25 \mathrm{~kg} / \mathrm{m}^{2}$ ), current smoking (no vs. yes), current drinking (no vs. yes), $\operatorname{SBP}(<140,140-159, \geq 160 \mathrm{mmHg}), \operatorname{DBP}(<90,90-99, \geq$ $100 \mathrm{mmHg}$ ), Diabetes mellitus (no vs. yes, and eGFR ( $<60 \mathrm{vs.} \geq 60 \mathrm{~mL} / \mathrm{min} / 1.73 \mathrm{~m}^{2}$ ).

Data were analyzed using the Empower (R;www.empowerstats.com; X\&Y Solutions, Inc, Boston, MA, USA) and the statistical package (R) (http://www.R-project.org, The R Foundation). Effects that met the 5\% significance level were considered statistically significant, with all tests were two-sided.

\section{Results}

\section{Baseline characteristics}

A representative sample of 4926 participants of the China Hypertension Registry study was eligible and enrolled in the analysis. From the study population, the mean age of those paticipants was $64.42 \pm 9.44$ years old, and 2440 (49.53\%) people were men. The overall mean baseline LAP index was 40.65 , ranging from 0.14 to 514.60 . And mean baPWV was $1855.27(414.58) \mathrm{cm} / \mathrm{s}$. The baseline clinical characteristics of participants according to the quartile of in LAP index are presented in Table 1. Compared with participants in the lowest quartile group, participants with a higher LAP index tended to be younger, women, and have diabetes mellitus and higher $\mathrm{BMI}$, and have a low prevalence of current smokers and drinkers, and have lower values of HDL, Hcy. Likewise, signifcant diferences in biological parameters were observed among the groups. DBP, pulse, FBG, TC, LDL, eGFR and uric acid of the participants in the highest LAP index quartile were signifcantly higher than those in the frst quartile. Moreover, the high LAP index groups had a higher use of antihypertensive drugs, antiplatelet drugs glucose-lowering drugs (all $P$ $<0.05)$. 
Table 1

Clinical characteristics of the study population according to LAP

\begin{tabular}{|c|c|c|c|c|c|}
\hline \multirow[t]{2}{*}{ Variable } & \multicolumn{4}{|l|}{ In LAP } & \multirow{2}{*}{$\begin{array}{l}P \\
\text { value }\end{array}$} \\
\hline & Quartile 1 & Quartile 2 & Quartile 3 & Quartile 4 & \\
\hline LAP range & 0.14 to $\otimes 14.75$ & $\begin{array}{l}14.75 \\
\text { to\29.79 }\end{array}$ & $\begin{array}{l}29.79 \\
\text { to } \ 52.83\end{array}$ & $\begin{array}{l}52.83 \text { to } \leq \\
514.60\end{array}$ & <. \\
\hline Participants & 1230 & 1233 & 1231 & 1232 & \\
\hline Males, N & 865 (70.33\%) & $620(50.28 \%)$ & $501(40.70 \%)$ & 454 (36.85\%) & $\begin{array}{l}< \\
0.001\end{array}$ \\
\hline Age,year & $67.47 \pm 8.81$ & $65.25 \pm 9.53$ & $63.85 \pm 9.20$ & $61.10 \pm 9.07$ & $<.001$ \\
\hline $\mathrm{BMI}, \mathrm{kg} / \mathrm{m}^{2}$ & $19.91 \pm 2.24$ & $22.60 \pm 2.35$ & $24.50 \pm 2.71$ & $26.12 \pm 3.08$ & $<.001$ \\
\hline $\begin{array}{l}\text { Smoking status, N } \\
(\%)\end{array}$ & $539(43.82 \%)$ & 331 (26.85\%) & $256(20.80 \%)$ & $262(21.27 \%)$ & $<.001$ \\
\hline $\begin{array}{l}\text { Drinking status, } N \\
(\%)\end{array}$ & 393 (31.95\%) & 294 (23.84\%) & $263(21.36 \%)$ & 266 (21.59\%) & $<.001$ \\
\hline SBP, mmHg & $\begin{array}{l}147.01 \pm \\
19.08\end{array}$ & $\begin{array}{l}147.22 \pm \\
17.56\end{array}$ & $\begin{array}{l}146.97 \pm \\
16.25\end{array}$ & $147.54 \pm 17.18$ & 0.850 \\
\hline $\mathrm{DBP}, \mathrm{mmHg}$ & $86.43 \pm 11.22$ & $88.86 \pm 10.99$ & $89.17 \pm 10.59$ & $91.15 \pm 10.43$ & $\begin{array}{l}< \\
0.001\end{array}$ \\
\hline pulse, bpm & $74.00 \pm 15.98$ & $75.44 \pm 14.85$ & $75.74 \pm 13.52$ & $78.49 \pm 13.92$ & $<.001$ \\
\hline $\mathrm{Hcy}, \mu \mathrm{mol} / \mathrm{L}$ & $19.55 \pm 11.63$ & $19.05 \pm 12.77$ & $17.90 \pm 11.00$ & $17.41 \pm 10.77$ & <. \\
\hline FBG, mmol/L & $5.69 \pm 1.06$ & $5.91 \pm 1.13$ & $6.17 \pm 1.69$ & $6.72 \pm 2.11$ & $<.001$ \\
\hline $\mathrm{TC}, \mathrm{mmol} / \mathrm{L}$ & $4.79 \pm 1.00$ & $5.02 \pm 1.02$ & $5.28 \pm 1.06$ & $5.48 \pm 1.19$ & $<.001$ \\
\hline $\mathrm{HDL}-\mathrm{C}, \mathrm{mmol} / \mathrm{L}$ & $1.71 \pm 0.44$ & $1.53 \pm 0.37$ & $1.43 \pm 0.33$ & $1.31 \pm 0.33$ & $\begin{array}{l}< \\
0.001\end{array}$ \\
\hline LDL-C, mmol/L & $2.50 \pm 0.65$ & $2.83 \pm 0.70$ & $3.13 \pm 0.74$ & $3.31 \pm 0.81$ & $<.001$ \\
\hline \multicolumn{6}{|c|}{$\begin{array}{l}\text { Abbreviation: BMI, body mass index; SBP, systolic blood pressure; DBP, diastolic blood pressure; CHD, } \\
\text { coronary heart disease; FBG: fasting blood glucose; Hcy, homocysteine; HDL-C, high-density } \\
\text { lipoprotein cholesterol; TC, total cholesterol; LDL-C, low-density lipoprotein cholesterol; eGFR, } \\
\text { estimated glomerular filtration rate. }\end{array}$} \\
\hline $\begin{array}{l}\text { \$diabetes mellitus } \\
\geq 7.0 \mathrm{mmol} / \mathrm{L} \text { or } \mathrm{u}\end{array}$ & $\begin{array}{l}\text { lefined as se } \\
\text { glucose-lowe }\end{array}$ & $\begin{array}{l}\text { ported physici } \\
\text { drugs. }\end{array}$ & iagnosis of & tes or FBG cc & ation \\
\hline
\end{tabular}




\begin{tabular}{|c|c|c|c|c|c|}
\hline \multirow[t]{2}{*}{ Variable } & \multicolumn{4}{|l|}{ In LAP } & \multirow{2}{*}{$\begin{array}{l}P \\
\text { value }\end{array}$} \\
\hline & Quartile 1 & Quartile 2 & Quartile 3 & Quartile 4 & \\
\hline Uric acid, mmol/L & $\begin{array}{l}416.46 \pm \\
115.47\end{array}$ & $\begin{array}{l}416.62 \pm \\
116.96\end{array}$ & $\begin{array}{l}430.02 \pm \\
120.91\end{array}$ & $\begin{array}{l}463.09 \pm \\
124.52\end{array}$ & $\begin{array}{l}< \\
0.001\end{array}$ \\
\hline $\begin{array}{l}\text { eGFR, mL/min/1.73 } \\
\mathrm{m}^{2}\end{array}$ & $83.93 \pm 19.91$ & $85.24 \pm 19.73$ & $87.37 \pm 18.68$ & $88.55 \pm 19.24$ & $\begin{array}{l}<.001 \\
0.001\end{array}$ \\
\hline Diabetes mellitus\$ & $108(8.78 \%)$ & 175 (14.19\%) & $236(19.17 \%)$ & $376(30.52 \%)$ & $\begin{array}{l}< \\
0.001\end{array}$ \\
\hline $\begin{array}{l}\text { Antihypertensive } \\
\text { drugs }\end{array}$ & 678 (55.12\%) & 743 (60.26\%) & 763 (61.98\%) & 787 (63.88\%) & $\begin{array}{l}< \\
0.001\end{array}$ \\
\hline Antiplatelet drugs & $16(1.30 \%)$ & $35(2.84 \%)$ & $33(2.68 \%)$ & $24(1.95 \%)$ & 0.034 \\
\hline $\begin{array}{l}\text { Glucose-lowering } \\
\text { drugs }\end{array}$ & $22(1.79 \%)$ & $40(3.24 \%)$ & $58(4.71 \%)$ & $81(6.57 \%)$ & $\begin{array}{l}< \\
0.001\end{array}$ \\
\hline \multicolumn{6}{|c|}{$\begin{array}{l}\text { Abbreviation: BMI, body mass index; SBP, systolic blood pressure; DBP, diastolic blood pressure; CHD, } \\
\text { coronary heart disease; FBG: fasting blood glucose; Hcy, homocysteine; HDL-C, high-density } \\
\text { lipoprotein cholesterol; TC, total cholesterol; LDL-C, low-density lipoprotein cholesterol; eGFR, } \\
\text { estimated glomerular filtration rate. }\end{array}$} \\
\hline
\end{tabular}

\section{Association of LAP index with baPWV and elevated baPWV}

Overall, the LAP index was found to be positive associated with baPWV in models $1-3$. Values are the regression coefficients $(\beta)$ of association, with model 1 adjusted for age, sex; model 2 adjusted for age, sex, BMI, SBP, DBP, pulse, smoking status, and drinking status; model 3 adjusted for age, sex, BMI, SBP, DBP, pulse, smoking status, drinking status. FBG, TC, HDL, LDL-C, Hcy, uric acid, eGFR, diabetes mellitus, antihypertensive drugs, and antiplatelet drugs. In models $1-3$, according to the $\beta$ coefficients, for every 1 unit increase in In LAP, the increases in baPWV were $19.81 \mathrm{~cm} / \mathrm{s}(95 \% \mathrm{Cl}: 9.51,30.11 \mathrm{~cm} / \mathrm{s}), 42.39 \mathrm{~cm} / \mathrm{s}$ (95\%Cl: $30.56,54.22 \mathrm{~cm} / \mathrm{s}$ ) and $25.06 \mathrm{~cm} / \mathrm{s}(95 \% \mathrm{Cl}: 10.91,39.22 \mathrm{~cm} / \mathrm{s})$, respectively. As possible confounding factors were removed the association became greater. We also converted In LAP from a continuous variable to a quartiles variable (Table 2). Compared with participants in the first quartile group, participants in quartile 2, quartile 3 , and quartile 4 of the LAP index tended to have increased $\beta$ coefficients of baPWV (all P-trend < 0.001 ). 
Table 2

The association between LAP and baPWV in diferent models

\begin{tabular}{|c|c|c|c|}
\hline \multirow[t]{2}{*}{ LAP Index } & \multicolumn{3}{|l|}{$\mathrm{BaPWV}, \mathrm{cm} / \mathrm{s}, \beta(95 \% \mathrm{Cl})$} \\
\hline & Model 1 & Model 2 & Model 3 \\
\hline Per 1 unit increase & $19.81(9.51,30.11)$ & $42.39(30.56,54.22)$ & $25.06(10.91,39.22)$ \\
\hline \multicolumn{4}{|l|}{ Quartiles } \\
\hline Q1 (0.14 to $ه 14.75)$ & 0 & 0 & 0 \\
\hline Q2 (14.75 to囚29.79) & $46.38(16.63,76.14)$ & $81.62(54.37,108.86)$ & $70.29(42.00,98.59)$ \\
\hline Q3 (29.79 to $₫ 52.83)$ & $29.99(-0.35,60.33)$ & $103.14(72.52,133.76)$ & $84.00(50.29,117.72)$ \\
\hline Q4 (52.83 to $\leq 514.60)$ & $75.90(44.75,107.04)$ & $150.06(115.80,184.32)$ & $109.96(69.73,150.18)$ \\
\hline$P$ for trend & $<0.001$ & $<0.001$ & $<0.001$ \\
\hline \multicolumn{4}{|c|}{ Model 1 was adjusted for age, sex. } \\
\hline \multicolumn{4}{|c|}{ Model 2 was adjusted for age, sex, BMI, SBP, DBP, pulse, smoking status, drinking status. } \\
\hline $\begin{array}{l}\text { Model } 3 \text { was adjusted } f \\
\text { HDL, LDL-C, Hcy, uric ac }\end{array}$ & סיח סיב & omoling ctatur dri & $\begin{array}{l}\text { ig status. FBG, TC, } \\
\text { platelet drugs. }\end{array}$ \\
\hline
\end{tabular}

As shown in Table 3, in a fully adjusted model, a positive association between the LAP and the risk of elevated baPWV was still exists. For every 1 -unit increase in InLAP, the adjusted ORs of elevated baPWV for participants in models $1-3$ were 1.17 (95\% Cl: 1.09, 1.25), 1.42( $95 \% \mathrm{Cl}: 1.28,1.58)$ and 1.24 (95\% Cl: $1.09,1.40)$, respectively. When we converted In LAP from a continuous variable to a quartiles variable, the adjusted OR of elevated baPWV for participants in quartile2- 4 was 1.51 (95\% Cl:1.18, 1.93), 1.80 (95\% Cl: $1.33,2.44)$, and 2.24 (95\% Cl:1.56, 3.21) compared with participants in quartile 1 ( $P$-trend $<0.001)$. A generalized additive model and penalized spline method was used further analyses the dose-response relation between InLAP and baPWV and elevated baPWV (Fig. 1A-1B), showing that there were signifcant positive linear association of LAP index with baPWV and elevated baPWV. 
Table 3

The association between LAP and elevated baPWV in diferent models

\begin{tabular}{|c|c|c|c|}
\hline \multirow[t]{2}{*}{ LAP Index } & \multicolumn{3}{|c|}{ Elevated baPWV, OR (95\%Cl) } \\
\hline & Model1 & Model 2 & Model 3 \\
\hline Per 1 unit increase & $1.17(1.09,1.25)$ & $1.42(1.28,1.58)$ & $1.24(1.09,1.40)$ \\
\hline \multicolumn{4}{|l|}{ Quartiles } \\
\hline Q1 (0.14 to $₫ 14.75)$ & 1 & 1 & 1 \\
\hline Q2 (14.75 toه29.79) & $1.27(1.04,1.55)$ & $1.66(1.31,2.10)$ & $1.51(1.18,1.93)$ \\
\hline Q3 (29.79 to $₫ 52.83)$ & $1.26(1.02,1.54)$ & $2.16(1.65,2.83)$ & $1.80(1.33,2.44)$ \\
\hline Q4 (52.83 to $\leq 514.60)$ & $1.71(1.38,2.11)$ & $3.14(2.32,4.26)$ & $2.24(1.56,3.21) 1$ \\
\hline$P$ for trend & $<0.001$ & $<0.001$ & $<0.001$ \\
\hline \multicolumn{4}{|c|}{ Model 1 was adjusted for age, sex. } \\
\hline
\end{tabular}

\section{Subgroup analyses by potential effect modifers}

To assess the relationship between In LAP (per 1 unit increment) and baPWV in various groups, we performed exploratory subgroup analyses (Fig. 2). There were no significant interactions in the following of the subgroup variables included sex (male vs. Female; $P$-interaction $=0.808)$, age $(<65$ vs. $\geq 65$ years; $P$ interaction $=0.587$ ), BMl (<25 vs. $\geq 25 \mathrm{~kg} / \mathrm{m}^{2} ;$ P-interaction $\left.=0.568\right)$, current smoking (no vs. yes; $P$ interaction $=0.061$ ), current drinking (no vs. yes; $P$-interaction $=0.861), \operatorname{SBP}(<140,140-159, \geq$ $160 \mathrm{mmHg} ;$ P-interaction $=0.283), \operatorname{DBP}(<90,90-99, \geq 100 \mathrm{mmHg} ; P$-interaction $=0.550)$, Diabetes mellitus (no vs. yes; $P$-interaction $=0.894)$, and eGFR $\left(<60 \mathrm{vs.} \geq 60 \mathrm{~mL} / \mathrm{min} / 1.73 \mathrm{~m}^{2} ; P\right.$-interaction $=$ 0.366). After adjustment for age, sex, BMI, SBP, DBP, pulse, smoking status, drinking status. FBG, TC, HDL, LDL-C, Hcy, uric acid, eGFR, diabetes mellitus, antihypertensive drugs, antiplatelet drugs, except for the stratifying variables (all $P$ for interactions $>0.05$ ).

\section{Discussion}

The current study includes 4926 participants, which is the largest number of research on the same subject so far. In this relatively large-scale cross-sectional study, we found for the first time that LAP index is independently positively correlated with baPWV and elevated baPWV in Chinese hypertensive patients, and no significant interaction was found in other subgroups. 
The relation of LAP index with baPWV have been evaluated in several previous studies and yield inconsistent results. Previous study has reported that LAP index in relation with the risk of arterial stiffness $[15,17]$.whereas some studies suggested that there was no association between LAP index and arterial stiffness[16]. Cicero et al.[15]conducted a study using the Brisighella Heart Study Database; A total of 1731 healthy adults were included in this study, The mean age was $58.7 \pm 15.8$ years and $58.4 \pm$ 15.5 years for men and women, respectively $(p=0.777)$. And the results showed that LAP was significantly correlated with the increase of bapWV $(R R=0.014,95 \% \mathrm{Cl} 0.008-0.020, p<0.001)$. A Japanese study explored the relationship between surrogate markers of insulin resistance including LAP and arteriosclerosis risk, including 1720 male (the mean age:38.8 \pm 10.1 years) and 1098 female (the mean age: $39.1 \pm 9$.4years) healthy adults. The results show that LAP is superior to other insulin resistance indexes including TyG, and LAP index is positively correlated with the elevated bapwv, which is more significant in women[17]. Wakabayashi et al[16]. included 954 Japanese health examination subjects aged 39-64, and found that LAP index was not related to the increased risk of arterial stiffness.The reasons for the inconsistency of the above research results may be related to the characteristics of the study population, the size of the sample and the definition of arterial stiffness. This study included 4926 participants with an average age of $64.42 \pm 9.44$ years, the results indicated that LAP index is positively correlated with bapwv and elevated baPWV, and there is no interaction between LAP index and baPWV in gender subgroup. Sex and menopause-related changes in body composition and lipid metabolism[22, 23], which may lead to differences in the significance of surrogate markers of insulin resistance related to gender and menopause may explain this finding. The women in this study population are basically in postmenopausal state, so the prediction ability of LAP index of women may decrease under estrogen level, which is similar to that of men. However, further research is needed to solve this hypothesis.

The exact mechanism of LAP index and arterial stiffness, especially in populations with hypertension, remains unclear. Studies have shown that LAP has a good correlation with insulin resistance and is considered as a surrogate marker of insulin resistance (IR) $[24,25]$. IR is closely related to the occurrence and development of atherosclerotic and is a risk factor of CVD[26]. The specific pathological mechanism is as follows: it is generally believed that plasma insulin has a beneficial effect on vascular endothelial function[27], and vasodilation is induced by nitric oxide production accelerated by insulin receptor through phosphatidylinositol 3- kinase (PI3k)/Akt insulin signaling pathway[28]. On the contrary, insulin resistance leads to the decrease of NO production by impairing PI3K/Akt signaling pathway[29], which is consistent with previous research results, which shows that insulin resistance is related to vascular dysfunction[30]. The effect of insulin resistance on arterial stiffness include that increase of sympathetic activity and the activation of renin-angiotensin-aldosterone system. IR can make the body in subclinical stress state, start the immune system slowly and slightly, and induce sustained and slight chronic inflammation, which leads to arterial stiffness[31, 32].

In order to better interpret the results of this study, we should include the limitations. First of all, this study is an cross-sectional study, so the causal relationship between LAP and baPWV cannot be obtained. Second, other confounding variables may affect our results, but regression analysis has adjusted a large 
number of main parameters, so we are trustful in our observation. Finally, the population of this study is the relatively old hypertensive population in China, so it is limited to generalizability the results of this study to other populations.

\section{Conclusions}

In summary, this large-scale cross-sectional study indicated that a signifcant positive association between LAP index and baPWV was found in Chinese adults with hypertension. In daily clinical practice, monitoring the LAP index deserves more attention for the early prevention of arterial stifness progressionin hypertensive patients.

\section{Declarations}

\section{Funding}

This work was supported by the National Natural Science Foundation of China (81760049), the Jiangxi Science and Technology Innovation Platform Project (20165BCD41005), the National Key R\&D Program of China (2018YFC1312902), the Key Project of Education Department of Jiangxi Province (GJJ170013).

\section{Conflict of Interests}

The authors declare that they have no conflict of interest.

\section{Ethical approval}

All procedures performed in studies involving human participants were following the ethical standards of the institutional and national research committee and with the 1964 Helsinki declaration and its later amendments or comparable ethical standards.

\section{Authors' contributions}

YMS participated in the literature search, data analysis, and data interpretation. YMS wrote the manuscript. LHH extracted and collected data. LHH, MHL, WZ, TW, LJZ HHB conceived of the study and participated in its design and coordination. LP and XSC participated in the study design and provided critical revision. All authors read and approved the final manuscript.

\section{Acknowledgements}

Thanks to all the investigators and subjects who participated in the China Hypertension Registry Study.

\section{Availability of data and materials}

The datasets used and/or analyzed in the current study are available from the corresponding author upon reasonable request. 


\section{Consent for publication}

Not applicable.

\section{References}

1. Williams B, Mancia G, Spiering W, Agabiti Rosei E, Azizi M, Burnier M, Clement DL, Coca A, de Simone G, Dominiczak A. et al: 2018 ESC/ESH Guidelines for the management of arterial hypertension: The Task Force for the management of arterial hypertension of the European Society of Cardiology and the European Society of Hypertension: The Task Force for the management of arterial hypertension of the European Society of Cardiology and the European Society of Hypertension. J Hypertens 2018, 36(10):1953-2041.

2. Satoh H, Kishi R, Tsutsui H. Metabolic syndrome is a significant and independent risk factor for increased arterial stiffness in Japanese subjects. Hypertens Res 2009, 32(12):1067-1071.

3. Ohkuma T, Ninomiya T, Tomiyama H, Kario K, Hoshide S, Kita Y, Inoguchi T, Maeda Y, Kohara K, Tabara Y. et al: Brachial-Ankle Pulse Wave Velocity and the Risk Prediction of Cardiovascular Disease: An Individual Participant Data Meta-Analysis. Hypertension 2017, 69(6):1045-1052.

4. Laurent S, Boutouyrie P. Arterial stiffness and stroke in hypertension: therapeutic implications for stroke prevention. CNS Drugs 2005, 19(1):1-11.

5. Sheng CS, Li Y, Li LH, Huang QF, Zeng WF, Kang YY, Zhang L, Liu M, Wei FF, Li GL. et al: Brachialankle pulse wave velocity as a predictor of mortality in elderly Chinese. Hypertension 2014, 64(5): 1124-1130.

6. Tomiyama H, Tanaka H, Hashimoto H, Matsumoto C, Odaira M, Yamada J, Yoshida M, Shiina K, Nagata $M$, Yamashina A. Arterial stiffness and declines in individuals with normal renal function/early chronic kidney disease. Atherosclerosis 2010, 212(1):345-350.

7. Ueshima H, Zhang XH, Choudhury SR. Epidemiology of hypertension in China and Japan. J Hum Hypertens 2000, 14(10-11):765-769.

8. Lee SJ, Avolio A, Seo DC, Kim BS, Kang JH, Lee MY, Sung KC. Relationship Between Brachial-Ankle Pulse Wave Velocity and Incident Hypertension According to 2017 ACC/AHA High Blood Pressure Guidelines. J Am Heart Assoc 2019, 8(16):e013019.

9. Wang Z, Chen Z, Zhang L, Wang X, Hao G, Zhang Z, Shao L, Tian Y, Dong Y, Zheng C et al: Status of Hypertension in China: Results From the China Hypertension Survey, 2012-2015. Circulation 2018, 137(22):2344-2356.

10. Guo QH, Zhang YQ, Wang JG. Asian management of hypertension: Current status, home blood pressure, and specific concerns in China. J Clin Hypertens (Greenwich) 2020, 22(3):475-478.

11. Kahn HS, Valdez R. Metabolic risks identified by the combination of enlarged waist and elevated triacylglycerol concentration. Am J Clin Nutr 2003, 78(5):928-934.

12. Kahn HS. The "lipid accumulation product" performs better than the body mass index for recognizing cardiovascular risk: a population-based comparison. BMC Cardiovasc Disord 2005, 5:26. 
13. Kahn HS. The lipid accumulation product is better than BMI for identifying diabetes: a populationbased comparison. Diabetes Care 2006, 29(1):151-153.

14. Nascimento-Ferreira MV, Rendo-Urteaga T, Vilanova-Campelo RC, Carvalho HB, da Paz Oliveira G, Paes Landim MB, Torres-Leal FL. The lipid accumulation product is a powerful tool to predict metabolic syndrome in undiagnosed Brazilian adults. Clin Nutr 2017, 36(6):1693-1700.

15. Fatty liver index is associated to pulse wave velocity in healthy subjects: Data from the Brisighella Heart Study. Eur J Intern Med2018, 53:29-33..

16. Wakabayashi I, Marumo M, Kubota Y, Higashiyama A, Miyamoto Y, Okamura T. Cardiometabolic index as a useful discriminator for the risk of increased arterial stiffness. Clin Chim Acta 2018, 486:42-43.

17. Nakagomi A, Sunami Y, Kawasaki Y, Fujisawa T, Kobayashi Y. Sex difference in the association between surrogate markers of insulin resistance and arterial stiffness. J Diabetes Complications 2020, 34(6): 107442.

18. Yu Y, Hu L, Huang X, Zhou W, Bao H, Cheng X. BMI modifies the association between serum HDL cholesterol and stroke in a hypertensive population without atrial fibrillation. J Endocrinol Invest 2021, 44(1):173-181.

19. Mousapour P, Barzin M, Valizadeh M, Mahdavi M, Azizi F, Hosseinpanah F: Predictive performance of lipid accumulation product and visceral adiposity index for renal function decline in non-diabetic adults, an 8.6-year follow-up. Clin Exp Nephrol 2020, 24(3):225-234..

20. Levey AS, Stevens LA, Schmid CH, Zhang YL, Castro AF 3rd, Feldman HI, Kusek JW, Eggers P, Van Lente F, Greene T. et al: A new equation to estimate glomerular filtration rate. Ann Intern Med 2009, 150(9):604-612.

21. Greenland S. Modeling and variable selection in epidemiologic analysis. Am J Public Health 1989, 79(3):340-349.

22. Geer EB, Shen W. Gender differences in insulin resistance, body composition, and energy balance. Gend Med 2009, 6Suppl 1(Supp/ 1):60-75.

23. Ley CJ, Lees B, Stevenson JC. Sex-and menopause-associated changes in body-fat distribution. Am J Clin Nutr 1992, 55(5):950-954.

24. Xia C, Li R, Zhang S, Gong L, Ren W, Wang Z, Li Q. Lipid accumulation product is a powerful index for recognizing insulin resistance in non-diabetic individuals. Eur J Clin Nutr 2012, 66(9):1035-1038.

25. Mazidi M. Surrogate markers of insulin resistance and arterial stiffness. J Diabetes Complications 2020, 34(6): 107491.

26. Reaven GM. Insulin resistance: from bit player to centre stage. CMAJ 2011, 183(5):536-537.

27. Mikhail N, Tuck ML. Insulin and the vasculature. Curr Hypertens Rep 2000, 2(2):148-153.

28. Zeng G, Nystrom FH, Ravichandran LV, Cong LN, Kirby M, Mostowski H, Quon MJ. Roles for insulin receptor, PI3-kinase, and Akt in insulin-signaling pathways related to production of nitric oxide in human vascular endothelial cells. Circulation 2000, 101(13):1539-1545. 
29. Kim JA, Montagnani M, Koh KK, Quon MJ. Reciprocal relationships between insulin resistance and endothelial dysfunction: molecular and pathophysiological mechanisms. Circulation 2006, 113(15): 1888-1904.

30. Hamburg NM, Larson MG, Vita JA, Vasan RS, Keyes MJ, Widlansky ME, Fox CS, Mitchell GF, Levy D, Meigs JB. et al: Metabolic syndrome, insulin resistance, and brachial artery vasodilator function in Framingham Offspring participants without clinical evidence of cardiovascular disease. Am J Cardiol 2008, 101(1):82-88.

31. Bornfeldt KE, Tabas I. Insulin resistance, hyperglycemia, and atherosclerosis. Cell Metab 2011, 14(5):575-585.

32. Ormazabal V, Nair S, Elfeky O, Aguayo C, Salomon C, Zuñiga FA. Association between insulin resistance and the development of cardiovascular disease. Cardiovasc Diabetol 2018, 17(1):122.

\section{Figures}
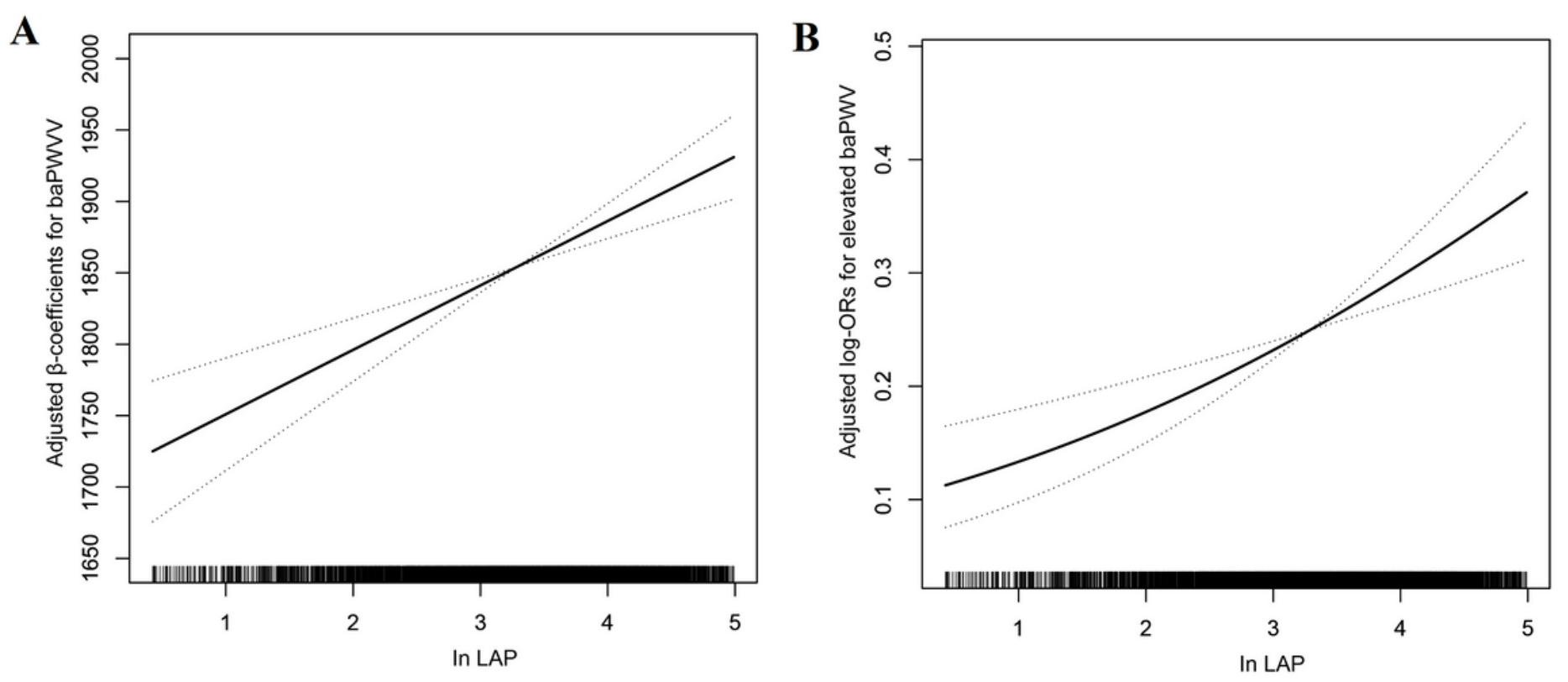

Figure 1

Association of LAP index with baPWV and elevated baPWV. A linear association between LAP index and baPWV and elevated baPWV was found $(P<0.05)$. The solid line and dashed line represent the estimated values and their corresponding 95\% confidence interval. Adjustment factors included age, sex, BMI, SBP, DBP, pulse, smoking status, drinking status. FBG, TC, HDL, LDL-C, Hcy, uric acid, eGFR, diabetes mellitus, antihypertensive drugs, antiplatelet drugs. 


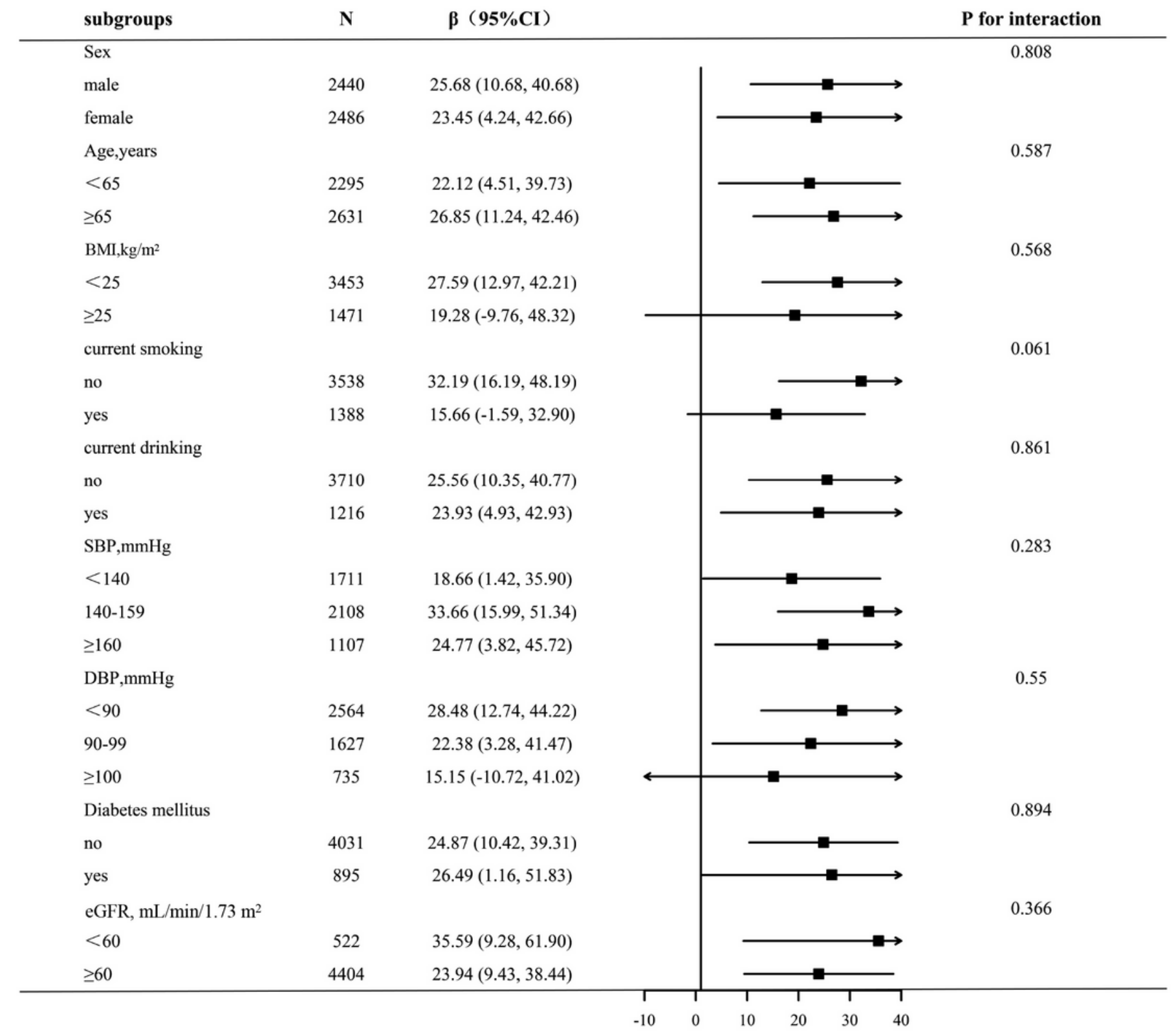

Figure 2

Subgroup analyses by potential effect modifers. *Each subgroup analysis adjusted for age, sex, BMI, SBP, DBP, pulse, smoking status, drinking status. FBG, TC, HDL, LDL-C, Hcy, uric acid, eGFR, diabetes mellitus, antihypertensive drugs, antiplatelet drugs. except for the stratifying variable.

\section{Supplementary Files}

This is a list of supplementary files associated with this preprint. Click to download.

- FigureS1.tif 